\title{
Pathos Vertex Semientire Graph of a Tree
}

\author{
Venkanagouda M. Goudar \\ Sri Gouthama Research centre (Affiliated to Kuvempu university), \\ Department of Mathematics, Sri Siddhartha Institute of Technology, \\ Tumkur, Karnataka- 572105. \\ Email: vmgouda@gmail.com
}

\begin{abstract}
In this communications, the concept of the pathos vertex semientire graph of a tree is introduced. We present characterization of graphs whose pathos vertex semientire graph of a tree is planar, outerplanar and minimally nonouterplanar. Also we establish a characterization of graphs whose pathos vertex semientire graph of a tree is noneularian , hamiltonian
\end{abstract}

Keywords: Innervertex number, Line graph, Outerplanar, Pathos, Pathoslength, Vertex semientire graph.

\section{Introduction}

By graph, we mean a finite, undirected graph without loops or multiple edges. We refer the terminology of [5].

The concept of pathos of a graph $\mathrm{G}$ was introduced by Harary[1] as a collection of minimum number of edge disjoint open paths whose union is $\mathrm{G}$. The path number of a graph $\mathrm{G}$ is the number of paths in the pathos. The path number of a tree $\mathrm{T}$ is equal to $\mathrm{k}$, where $2 \mathrm{k}$ is the number of odd degree vertices of $\mathrm{T}$. In addition, the end vertices of each path of any pathos of a tree are of odd degree.

The edgedegree of an edge uv of a tree $T$ is the sum of the degrees of $u$ and $v$. The pathoslength is the number of edges that lie on a particular path $\mathrm{P}_{i}$ of pathos of $\mathrm{T}$. A pendent pathos is a path $\mathrm{P}_{\mathrm{i}}$ of pathos having unit length, which corresponds to a pendent edge in $\mathrm{T}$. A pathosvertex is a vertex in $\mathrm{Pe}_{\mathrm{v}}(\mathrm{T})$ corresponding to a path of pathos of $\mathrm{T}$. A regionvertex is a vertex in $\mathrm{Pe}_{\mathrm{v}}(\mathrm{T})$ corresponding to a region of $\mathrm{T}$. 
The inner vertex number $i(G)$ of a planar graph $G$ is the minimum number of vertices not belonging to the boundary of the exterior region in any embedding of $\mathrm{G}$ in the plane. A graph $\mathrm{G}$ is said to be minimally nonouterplanar if $\mathrm{i}(\mathrm{G})=1$.

A new concept of a graph valued functions called the vertex semientire graph $e_{v}(G)$ of a plane graph $G$ was introduced by Kulli [4] and is defined as the graph whose vertex set can be put in one - to - one correspondence with the vertices and regions of $\mathrm{G}$ in such a way that two vertices of $e_{\mathrm{v}}(\mathrm{G})$ are adjacent if and only if the corresponding elements of $\mathrm{G}$ are adjacent.

The pathos vertex semientire graph of a tree denoted by $\mathrm{Pe}_{\mathrm{v}}(\mathrm{T})$ is the graph whose vertex set is $\mathrm{V}(\mathrm{T}) \mathrm{Y} \mathrm{P}_{\mathrm{i}} \mathrm{Yr}$ and the two vertices are adjacent if and only if they are adjacent vertices, vertices lie on the path of pathos and vertices lie on the regions. Since the system of pathos for a tree is not unique, the corresponding pathos vertex semientire graph of a tree is also not unique.

\section{Preliminaries}

We need the following results to prove further results.

Theorem 2.1 [Ref 4 ]: If $G$ be a connected plane graph then $e_{v}(G)$ is planar if and only if $G$ is a tree.

Theorem 2.2 [Ref 3]: Every maximal outerplanar graph $G$ with $p$ vertices has $2 p-3$ edges.

\section{Pathos Vertex Semientire Graph of a Tree}

We start with a preliminary result.

Remark 3.1: For ant tree $T, T \subseteq e_{v}(T) \subseteq P e_{v}(T)$.

In the following theorem we obtain the number of vertices and edges in a pathos vertex semientire graph of a tree.

Theorem 3.1: For any $(p, q)$ graph $T$ with $k$ path of pathos, $r$ regions, then pathos vertex semientire graph of a tree $P e_{v}(T)$ has $(p+k+1)$ vertices and

$$
p+q+\sum_{i=1}^{k} P_{i} \text { edges. }
$$

Proof.3.1: By the definition of pathos vertex semientire graph of a tree $\operatorname{Pe}_{\mathrm{v}}(\mathrm{T})$, the number of vertices is the union of the vertices, path of pathos and the region 
vertices of $\mathrm{T}$ as the tree contain one region. Hence the number of vertices of pathos vertex semientire graph of a tree $\operatorname{Pe}_{\mathrm{v}}(\mathrm{T})$ is $(\mathrm{p}+\mathrm{k}+1)$.

Further, by remark 1, tree $\mathrm{T}$ is a sub graph of $\mathrm{Pe}_{\mathrm{v}}(\mathrm{T})$, hence degree of regionvertex is the number of vertices of T. Also the degree of pathosvertex $\mathrm{P}_{\mathrm{i}}$ is the number of vertices which lies on the path of pathos, which is $\sum_{i=1}^{k} P_{i}$. Hence the number of vertices of $\operatorname{Pe}_{\mathrm{v}}(\mathrm{T})=\mathrm{q}+\mathrm{p}+\sum_{i=1}^{k} P_{i}$.

Theorem 3.2: For any tree $T$, pathos vertex semientire graph of a tree $P e_{v}(T)$ is always planar.

Proof. 3.2: By the Theorem 1, vertex semientire graph of a tree $e_{v}(T)$ is always planar. By the definition of pathos vertex semientire graph of a tree $\operatorname{Pe}_{\mathrm{v}}(\mathrm{T})$, the regionvertex $r$ is adjacent to all vertices of $T$ and the degree of pathosvertex $P_{i}$ is the number of vertices which lies on the path of pathos of $\mathrm{T}$. Clearly this pathosvertex does not loose their planarity. Hence pathos vertex semientire graph of a tree $\operatorname{Pe}_{\mathrm{v}}(\mathrm{T})$ is always planar.

Theorem 3.3.: For any tree $T$ the pathos vertex semientire graph of a tree $P e_{v}(T)$ outerplanar if and only if $T$ is a path $P_{2}$.

Proof. 3.3: Suppose pathos vertex semientire graph of a tree $\operatorname{Pe}_{v}(T)$ is outerplanar. Assume that a tree $\mathrm{T}$ is not $\mathrm{P}_{2}$. If a tree $\mathrm{T}$ is $\mathrm{P}_{3}$, then by the definition of $\mathrm{Pe}_{\mathrm{v}}(\mathrm{T}), \mathrm{Pe}_{\mathrm{v}}\left(\mathrm{P}_{3}\right)=\mathrm{W}_{1,4}$, which is nonouterplanar, a contradiction. In general $\mathrm{Pe}_{\mathrm{v}}(\mathrm{T})$ contains $\mathrm{W}_{1, \mathrm{n}}$ as a sub graph, which is nonouterplanar. Hence $\mathrm{T}$ must be $\mathrm{P}_{2}$. Conversely, suppose $\mathrm{T}$ is $\mathrm{P}_{2}$. By definition of $\mathrm{Pe}_{\mathrm{v}}(\mathrm{T})$, the regionvertex $r$ is adjacent to two vertices $v_{1}, v_{2}$ to form $K_{3}$ and a pathosvertex $P_{i}$ is adjacent to two vertices $\mathrm{v}_{1}, \mathrm{v}_{2}$ fo $\mathrm{K}_{3}$ to form $\mathrm{K}_{4}-\mathrm{x}$, which is outerplanar.

Theorem 3.4.: For any tree $T$, pathos vertex semientire graph of a tree $P e_{v}(T)$ is maximal outerplanar if and only if $T$ is a path $P_{2}$.

Proof. 3.4: Suppose, pathos vertex semientire graph of a tree $\mathrm{Pe}_{\mathrm{v}}(\mathrm{T})$ is maximal outerplanar, then $\mathrm{Pe}_{\mathrm{v}}(\mathrm{T})$ is connected. Let $\mathrm{T}$ be a path $\mathrm{P}_{2}$, it contains $\mathrm{p}=2$ vertices and $\mathrm{q}=1$ edge. Given $\mathrm{Pe}_{\mathrm{v}}(\mathrm{T})$ is maximal outerplanar, by Theorem 2, if has $2 \mathrm{p}-3$ edges. By the definition of $\mathrm{Pe}_{\mathrm{v}}(\mathrm{T}), \mathrm{V}\left[\mathrm{Pe}_{\mathrm{v}}\left(\mathrm{P}_{2}\right)\right]=4$ and $\mathrm{E}\left[\mathrm{Pe}_{\mathrm{v}}(\mathrm{T})\right]=5$. 
$\Rightarrow 2 p-3=q$

$\Rightarrow 2 X 4-3=5$

$5=5$ is satisfied.

Clearly, $\mathrm{T}=\mathrm{P}_{2}$ is a nonempty path. Hence necessity is proved.

Theorem 3.5: For any tree $T$, pathos vertex semientire graph of a tree $P e_{v}(T)$ is minimally nonouterplanar if and only if $T$ is a path $P_{3}$.

Proof. 3.5: Suppose pathos vertex semientire graph of a tree $\operatorname{Pe}_{v}(T)$ is minimally nonouterplanar. Assume that a tree $\mathrm{T}$ is not a path $\mathrm{P}_{3}$. Let $\mathrm{T}=\mathrm{P}_{4}$, by the definition of pathos vertex semientire graph of a tree $\operatorname{Pe}_{v}(T)$, the regionvertex $r_{1}$ is adjacent to four vertices $\mathrm{v}_{1}, \mathrm{v}_{2}, \mathrm{v}_{3}, \mathrm{v}_{4}$. Also the pathosvertex is adjacent to these four vertices, each set $\left\{v_{1}, v_{2}, r_{1}, P_{1}\right\}$ forms an induced subgraph as $\mathrm{K}_{4}-\mathrm{x}$. Clearly $\mathrm{v}_{2}, \mathrm{v}_{3}$ are the inner vertices of $\operatorname{Pe}_{\mathrm{v}}(\mathrm{T})$, hence $\mathrm{i}\left[\mathrm{Pe}_{\mathrm{v}}\left(\mathrm{P}_{4}\right)\right]=2$, which is not minimally nonouterplanar, a contradiction.

Conversely, suppose a tree $\mathrm{T}$ is $\mathrm{P}_{3}$. By the theorem $5, \mathrm{Pe}_{\mathrm{v}}\left(\mathrm{P}_{3}\right)=\mathrm{W}_{1,4}$ and $\mathrm{i}\left[\mathrm{W}_{1,4}\right]=1$. Hence $\mathrm{Pe}_{\mathrm{v}}\left(\mathrm{P}_{3}\right)$ is minimally nonouterplanar.

Theorem 3.6: For any tree $T$, pathos vertex semientire graph of a tree $P e_{v}(T)$ is non Eulerian.

Proof. 3.6: Suppose a tree $T$ is a path $P_{n}: u_{1}, u_{2}, \ldots u_{n}, n>1$. Further $V\left[e_{v}(T)=\right.$ $\left\{\mathrm{u}_{1}, \mathrm{u}_{2}, \ldots \mathrm{u}_{\mathrm{n}}, \mathrm{r}_{1}\right\}$ where $\mathrm{r}_{1}$ is only one regionvertex. In $\mathrm{Pe}_{\mathrm{v}}(\mathrm{T})$ of a path $\mathrm{P}_{\mathrm{n}}$, each set $\left\{\mathrm{u}_{1}, \mathrm{u}_{2}, \mathrm{r}_{1}, \mathrm{P}_{1}\right\},\left\{\mathrm{u}_{2}, \mathrm{u}_{3}, \mathrm{r}_{1}, \mathrm{P}_{2}\right\}, \ldots\left\{\mathrm{u}_{\mathrm{n}-1}, \mathrm{u}_{\mathrm{n}}, \mathrm{r}_{1}, \mathrm{P}_{\mathrm{n}}\right\}$ forms an induced subgraph as $\mathrm{K}_{4}-\mathrm{x}$. Clearly $\operatorname{deg}\left(\mathrm{u}_{1}\right)=\operatorname{deg}\left(\mathrm{u}_{\mathrm{n}}\right)=$ odd in number. Hence $\mathrm{Pe}_{\mathrm{v}}(\mathrm{T})$ is nonEulerian.

Suppose a tree $\mathrm{T}$ is not a path. By the above case, the degree of pendent vertices is odd and hence $\mathrm{Pe}_{\mathrm{v}}(\mathrm{T})$ is nonEulerian. Hence $\mathrm{Pe}_{\mathrm{v}}(\mathrm{T})$ is always nonEulerian.

Theorem 3.7: For any tree T, pathos vertex semientire graph of a tree $P e_{v}(T)$ is always Hamiltonian.

Proof. 3.7: Suppose a tree $T$ is a path $P_{n}: u_{1}, u_{2}, \ldots u_{n}, n>1$. Clearly $T$ has exactly one path of pathos $\mathrm{P}_{1}$ and one regionvertex $\mathrm{r}_{1}$. Further $\mathrm{V}\left[\mathrm{e}_{\mathrm{v}}(\mathrm{T})=\left\{\mathrm{u}_{1}, \mathrm{u}_{2}, \ldots \mathrm{u}_{\mathrm{n}}, \mathrm{r}_{1}\right\}\right.$. Hence in $\mathrm{Pe}_{\mathrm{v}}(\mathrm{T})$ of a path $\mathrm{P}_{\mathrm{n}}$, each set $\left\{\mathrm{u}_{1}, \mathrm{u}_{2}, \mathrm{r}_{1}, \mathrm{P}_{1}\right\},\left\{\mathrm{u}_{2}, \mathrm{u}_{3}, \mathrm{r}_{1}, \mathrm{P}_{1}\right\}, \ldots\left\{\mathrm{u}_{\mathrm{n}-1}, \mathrm{u}_{\mathrm{n}}, \mathrm{r}_{1}, \mathrm{P}_{1}\right\}$ forms an induced subgraph as $\mathrm{K}_{4}-\mathrm{x}$. Clearly $\mathrm{r}_{1}, \mathrm{u}_{1}, \mathrm{P}_{1}, \mathrm{u}_{2}, \mathrm{u}_{3}, \ldots \mathrm{u}_{\mathrm{n}}, \mathrm{r}_{1}$ form a cycle containing all the vertices of $\mathrm{Pe}_{\mathrm{v}}(\mathrm{T})$. Hence $\mathrm{Pe}_{\mathrm{v}}(\mathrm{T})$ is Hamiltonian.

Also if a tree $T$ is not a path and contain $n$ vertices. Let $V\left[e_{v}(T)=\left\{u_{1}, u_{2}, \ldots u_{n}, r_{1}\right\}\right.$ where $r_{1}$ is only one regionvertex. In $\operatorname{Pe}_{v}(T)$, each set $\left\{r_{1}, u_{1}, u_{2}, P_{1}\right\},\left\{r_{1}\right.$, $\left.\mathrm{u}_{2}, \mathrm{u}_{3}, \mathrm{P}_{2}\right\}, \ldots\left\{\mathrm{r}_{1}, \mathrm{u}_{\mathrm{n}-1}, \mathrm{u}_{\mathrm{n}}, \mathrm{P}_{\mathrm{n}}\right\}$ forms an induced subgraph as $\mathrm{K}_{4}-\mathrm{x}$. Clearly these 
vertices $r_{1}, u_{1}, P_{1}, u_{2}, u_{3}, P_{2}, \ldots r_{1}$ form a cycle containing all the vertices of $\operatorname{Pe}_{v}(T)$ and it is Hamiltonian. Hence $\mathrm{Pe}_{\mathrm{v}}(\mathrm{T})$ is always Hamiltonian.

\section{References}

[1] Haray F., Annals of New York, Academy of Sciences, (1977)175, 198.

[2] Harrary, F.,Graph Theory, Addison- Wesley Reading Mass, (1969)., p.72, 107.

[3] Kulli. V R., On minimally nonouterplanar graphs, proceedings of the Indian National Science Academy, (1975)., 41A.

[4] Kulli. V. R. and Akka. D. G., Journal mathematical Science, Vol. 14, No. 6 , (1980)., P. 585- 588.

[5] Sedlacek J., Some properties of interchange graphs. The Graphs and the applications. Academic press, New York (1962).

[6] Y.B.Maralabhavi, Muddebihal and Venkanagouda. M. Goudar, "On Pathos semientire graph of a tree", in Far East Journal of Applied Mathematics, 27 (1) (2007).,pp. $85-91$. 